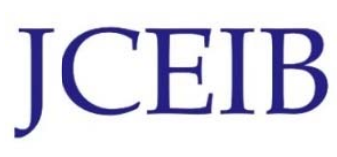

Journal Chemical Engineering and Industrial Biotechnology (JCEIB) Open Access

Volume 4 pp. 1-9; 30 September 2018

(C)Universiti Malaysia Pahang Publisher

DOI: https://doi.org/10.15282/JCEIB-V4-01.30/9/2018/4.4

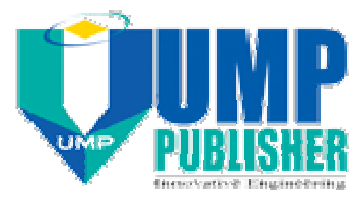

\title{
INCORPORATION OF HUMAN FACTORS IN RISK ANALYSIS OF OIL AND GAS PIPELINE USING BAYESIAN NETWORK
}

\author{
Fares Ahmed Alaw ${ }^{1}$, NurulSa'aadah Sulaiman ${ }^{1 *}$, Henry Tan ${ }^{2}$ \\ ${ }^{1}$ Faculty of Chemical and Natural Resources Engineering, Universiti Malaysia Pahang, \\ LebihrayaTunRazak, 26300Gambang,Kuantan, Pahang, Malaysia \\ ${ }^{2}$ School of Engineering, University of Aberdeen, Aberdeen AB24 3UE, United Kingdom. \\ *Corresponding author: E-mail: saaadah@ump.edu.my \\ Tel.: +601110033038
}

\begin{abstract}
Billions of barrels of oil and gas are consumed around the world daily and these oil and gas are being mainly transported and distributed through pipelines. The pipelines are demonstrably safe and are reliable systems to transport hydrocarbons, owing to the combination of good design, materials, and operating practices. However, if the pipeline fail, it is one of the most frustrating issues as its significant adverse would impact environment and public safety as well as severe economic loss. The objective of this study is to construct a cause and effect relationship framework of pipeline failure due to human factor using Bayesian Network (BN) approach. The potential human factors of the pipeline failure linked to corrosion were identified and categorized into three categories that are maintenance, monitoring, and operational errors. The predictive and diagnosis analyses of the Bayesian Network were performed to find the casual links which cause the failure in the system and make a prediction of the control measures to reduce the rate of the human mistakes. Results revealed that operational error showed a significant effect when the system operates beyond the limits of its design. In conclusion, Bayesian Networks appear to be a solution to build an effective oil and gas pipeline human error management model by providing information about the important human error that needs to be controlled. Thus, this framework may assist the decision maker to decide when and where to take preventive or mitigate measures in the risk management process of a pipeline.
\end{abstract}

Keywords: Human factors; Bayesian network; Oil and gas pipeline; Risk analysis.

\subsection{INTRODUCTION}

Oil and gas pipelines are a safe form of energy transportation, and the industry now has many years of operational experience. However, there continue to be frequent and tragic pipeline failures around the world (Hill et al., 2012). Leakage of oil and gas pipelines occurs due to the material defects, operational errors or other reasons, which may cause explosions and fires, resulting in casualties, environmental damage and material loss (Sulaiman and Tan, 2014). The safety of today's pipelines is dependent on not only their design and operation, but also their maintenance, and management (Khakzad et al., 2011). Therefore, it is important to develop new risk assessment technique that can provide more information and flexibility to the industry for better risk management than the available techniques. 
The causes of pipeline failures can range from internal issues such as corrosion or material defects to outside forces (Yang, 2010; Yang and Mannan, 2010). Human error is a very sensitive topic because it involves the actions of an individual (Hill et al., 2012). As one of the most challenging factors to be tracked down, human error factors are always related to many different areas, including engineering and psychology. To build a model to assess human error, engineers need to deal with many elements, such as identifying incorrect operation factors, defining the scope of the model, the methodology, designing, and data collection (Wu et al., 2015).

Risk assessment methods should take into account all these factors in combination with each other. Therefore, it can be stated that available methodologies are not able to provide accurate results because of their inadequate ability to describe the variety of risks. Hence, it is important to develop a method that has the ability to quantify risk arising from the uncertainty of the pipeline failure due to human factors. In this work, Bayesian network approach is introduced to determine and predict risk associated with human interventions.

A Bayesian network describes causal influence relations among variables via a directed acyclic graph. A probability is associated with each state of the node. This probability is defined, a priori for a root node and computed by inference for the others (Weber et al. 2012). Bayes' theorem provides a means for making these probability calculations. Essentially, it is a relationship between conditional probability $\mathrm{P}(\mathrm{A} \mid \mathrm{B})$ and marginal probabilities ( $\mathrm{P}(\mathrm{A})$ and $\mathrm{P}(\mathrm{B})$ ) and is given for two events, as illustrated in Figure 1, can be expressed by Bayes' Theorem:

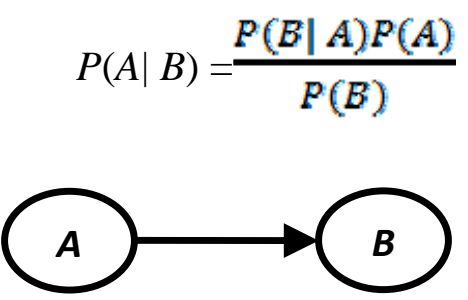

Figure 1: Basic example of a Bayesian Network

\subsection{MATERIALS AND METHODS}

Figure 2 summarises the steps to develop a Bayesian Network risk assessment model. The details of each step are described as follows:

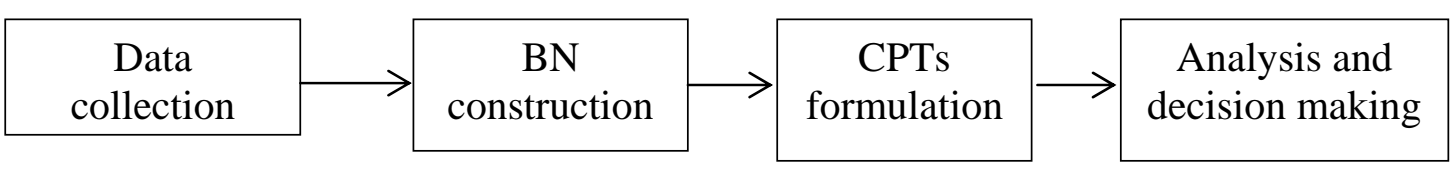

Figure 2: Flow diagram for risk assessment model

\subsection{Data collection}

The scope of risk analysis in this present paper is limited to corrosion damage. The goal of the proposed model was to estimate the probability of pipeline condition by taking into account the influence of human factors coming from various area. The areas are divided into three main categories for instance during maintenance, monitoring and operation activities. In data collection stage, the parameters that reflect the causality 
scenario were determined. Literature search or pipeline historical data due to corrosion is utilised throughout the understanding of the scenario.

\subsection{Development of Bayesian Network model}

The first step in constructing the $\mathrm{BN}$ is the development of the graphical representation to express the cause and effect relationships between the variables. This is important as it provides a straightforward means of analysing and communicating causal assumptions that are not easily expressed using standard mathematical notation. In this study, a commercial software package namely Hugin Expert was used to construct the BN model.Software Hugin Expert allows interactive creation of the network, maintenance of knowledge bases and integrates new evidence, efficient algorithm to support the implementation of Bayesian probability calculations, thus making a complete probabilistic model (Eleye-Datubo et al. 2006).

\subsection{Formulation of CPTs and prior probabilities}

The next step was to specify the possible states and define the conditional probability tables (CPTs) value. The data for prior probabilities and conditional probability tables were gathered from existing literatures. Prior to performing the analysis, the probability values in every column of CPTs were normalized to become 1 . Probability values of the marginal and conditional were required to be nonzero in which each condition of CPT is in the range of 0 to 1 . The information was collected from existing literatures for onshore pipeline incidents.

\subsection{Bayesian Network analyses}

Two types of analyses were carried out namely prediction analysis and diagnosis analysis. In the prediction analysis, the model will be updated whenever new knowledge or evidence is available. Meanwhile for the diagnostic analysis, the accidental path will be discovered and the posterior probability will be calculated. Based on the results obtained, a countermeasure to reduce the risk of the important factors were suggested in order to mitigate human errors.

\subsection{Proposed Bayesian Network model}

\subsection{RESULTS AND DISCUSSION}

The information was collected based on available literature. Referring to the available data in existing literature, the three main areas that human error can occur are during maintenance, monitoring and operation activities. The possible human errors for each category is summarised in Table 1. The developed Bayesian network the relationships of the potential human error at many points along the life cycle of a pipeline is shown in Figure 3. This life cycle provides an organization with the capability to integrate human factors into programs, standards, procedures, and process using a disciplined approach. In the category of maintenance error, the pipeline integrity condition usually being monitored and assessed by the determination of inspection frequency. Other than that, selection of the inspection tool used to evaluate the threat and tool capability were taking into account as these will effect on the inspection report. Operational error 
category is commonly caused by individual or organisational errors. Individual errors are those made by a particular person that contributes to an accident.The sources of organisational errors on the other hand, can be due to poor upper-level management and poor communication between the top level and the subordinates (Sulaiman, 2017). Finally monitoring errors, can also be observed with human-system interfaces such as equipment, software or instructions manual. These errors may eventually result in an operator making improper decisions.

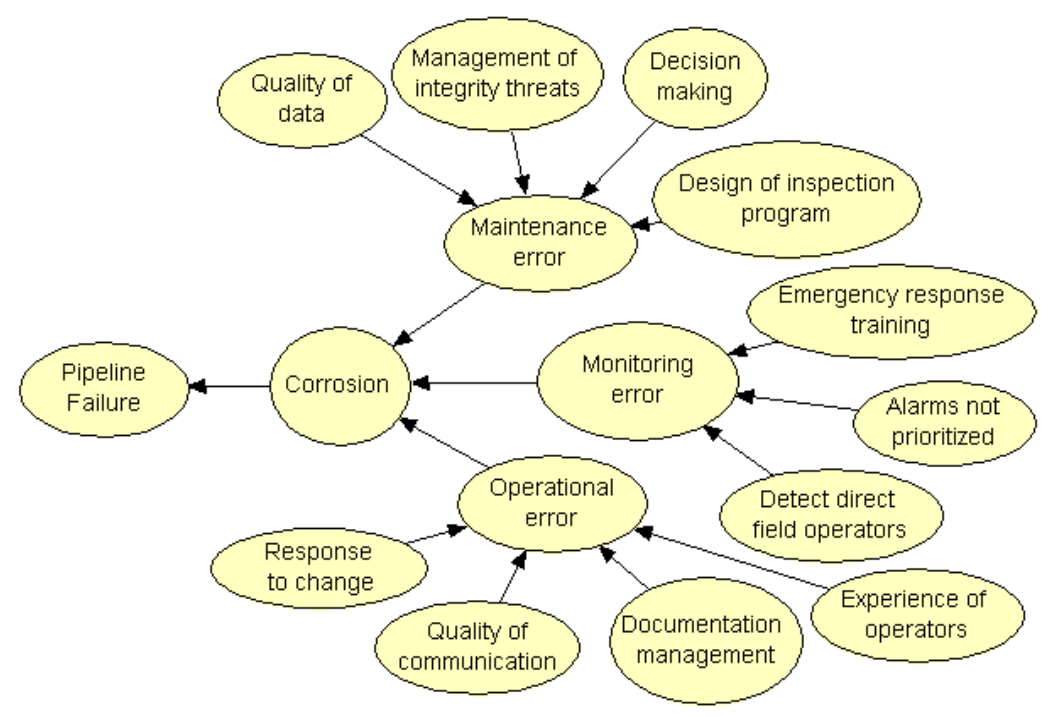

Figure 3: BN model of risk due to different errors.

\subsection{Prior probabilities generation}

The variables and states of activities on pipeline that will lead to error tabulated as in Table 1. In this paper, the probability data were extracted from different literature sources rather than from a specific pipeline. These data sources were gathered base on the experience from different pipeline operators in order to generate the prior probabilities and conditional probability tables.

Table 1: Parameters and the data sources for probability distributions.

\begin{tabular}{lll}
\hline Parameter & States & Probability data sources \\
\hline Corrosion and rupture & Yes, No & Shan et al. (2017) \\
Maintenance error & Yes, No & Adebayo et al. (2008) \\
Quality of data & Good, Poor & Adebayo et al. (2008) and Shan et al. (2017) \\
Management of threats & Good, Poor & Muhlbauer (2004) \\
Decision making & Good, Poor & Shan et al. (2017) and Muhlbauer (2004) \\
Design of inspection program & Good, Poor & Trucco et al. (2008) \\
Monitoring error & Yes, No & Adebayo et al. (2008) \\
Emergency response training & Good, Poor & Adebayo et al. (2008) and Revie (2015) \\
Alarms prioritized & Yes, No & Hill et al. (2012) \\
Detect and direct field operators & Good, Poor & Muhlbauer (2004) \\
Operation error & Yes, No & Adebayo et al. (2008) \\
Experience of operators & Good, Poor & Revie (2015) and Shan et al. (2017) \\
Documentation management & Good, Poor & El-Abbasy et al. (2015) \\
Quality of communication & Good, Poor & El-Abbasy et al. (2015) and Revie (2015) \\
Response to change & Good, Poor & Adebayo et al. (2008), Hill et al. (2012) and Revie \\
& & (2015) \\
\hline
\end{tabular}




\subsection{Bayesian Network analyses}

The prior probabilities from the data sources listed in Table 1 were utilised to determine the probability of pipeline failure. The prediction of the pipeline failure is as depicted in Figure 4. From the results obtained, the highest error found was from operational activities contributing to 56\%, which include experience of operators (65\%), documentation management (47\%), quality of communication (73\%), and response to change (39\%) are associated with pipeline failure.Operational errors occur when a system or process operates outside of or beyond the parameters of its design. For example, if specified operating practices call for a specific operating temperature, and a worker makes a decision to exceed this temperature, accelerated corrosion may be the result.

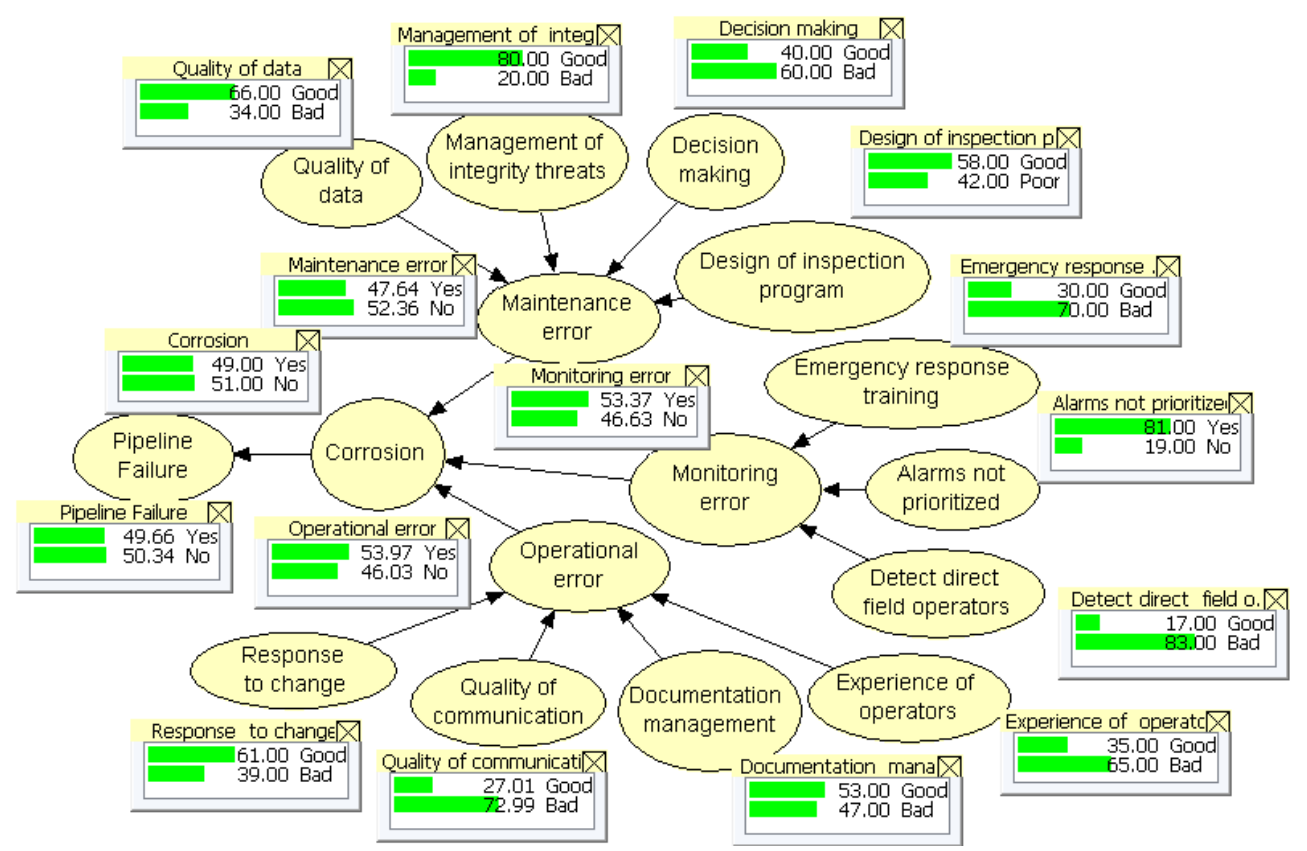

Figure 4: BN Model with Monitoring Window

The second highest error of 55\% contribution was from monitoring errors which consist of emergency response training (70\%), alarms prioritized (19\%), and direct field operators (83\%) caused pipeline failure via corrosion and/or rupture of the pipeline system. Monitoring errors are said to occur when a problem is noticed, but no action is taken. Often, a worker may believe that someone else will take care of the problem, or that it's someone else's responsibility.

Finally, maintenance error with $45 \%$ contributions which include quality of data (34\%), management of threats (20\%), decision making (60\%), and design of inspection program (42\%) will lead to pipeline failure. Maintenance errors occur when maintenance personnel fails to properly maintain or repair a system or improperly install one of its components.

\subsubsection{Diagnosis analysis}

The diagnostic analysis in Bayesian network inference was adopted to calculate the posterior probabilities of basic eventswhich can be used to find the weak links exist in the human error of oil and gas pipelines. The posterior probability distribution of each risk factor in case of an accident. 
In this initialised situation, the nodes were characterised by their prior probabilities as presented in Figure 4. Suppose it observed that Pipeline Failure is in state Yes and $\mathrm{P}($ Pipeline Failure $=Y e s)=1$ was entered into the model. This entered evidence increase the belief in all of the possible causes based on diagnostic inference. This will result in $57 \%$ probability due to maintenance, $66 \%$ due to monitoring and $68 \%$ due to operation errors. Figure 5 shows the results of the revised posterior probability for each risk factor. It can be observed that the occurrence probability due to maintenance error, monitoring error and operational error increase to approximately 57\%, 66\% and 68\% respectively.

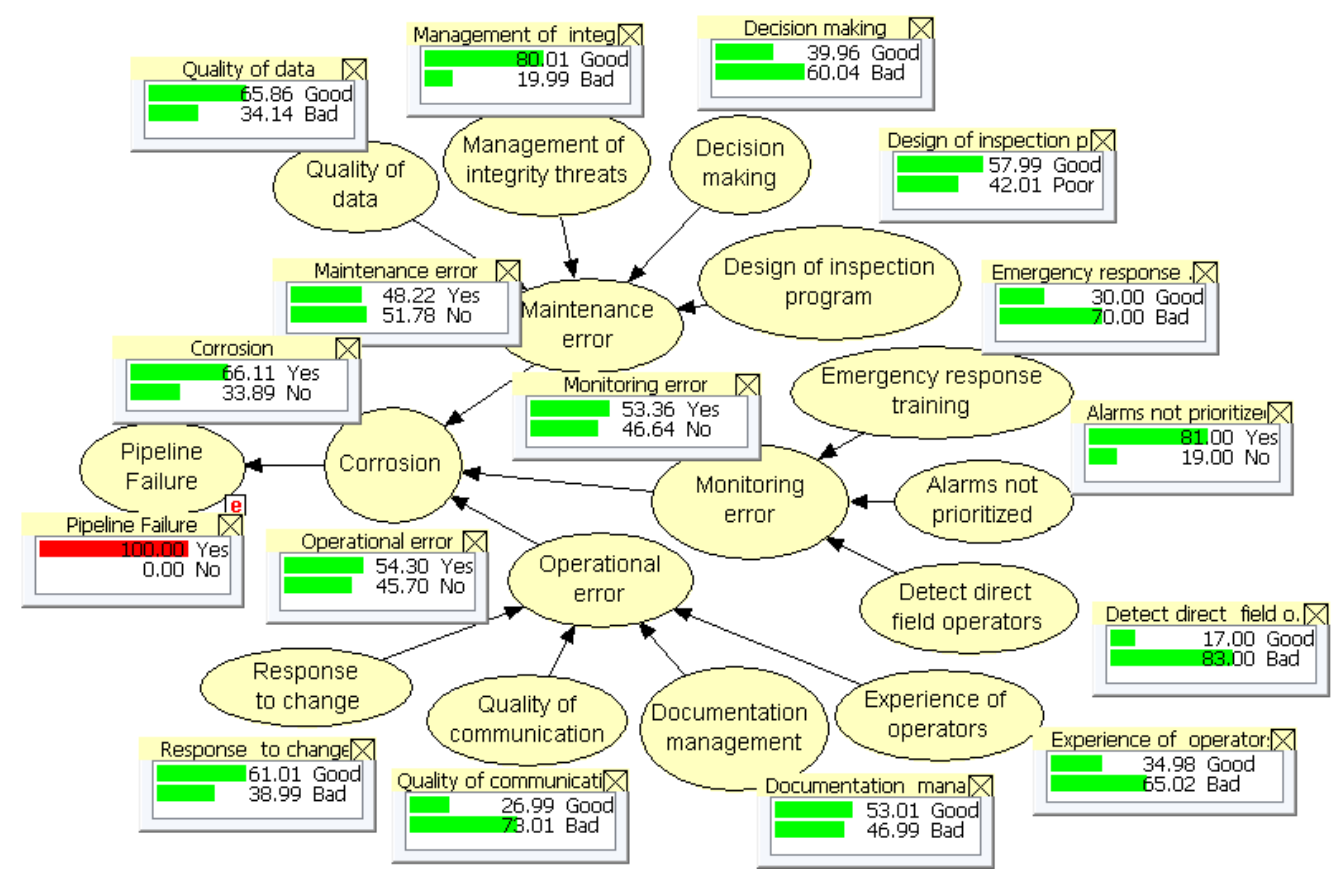

Figure 5: BN Model for Diagnosis Analysis

Overall the most influence factors are from bad decision making and poor communication in the organisational. From the analysis, the practical fault diagnosis and checking should then focus on the probability of these suspected factors to control the risk of pipeline failure. Therefore, the posterior probabilities can provide new evidential information for fault diagnosis in real time. By performing this analysis, the posterior joint probability of all variables/parents given the accident occurrence are helpful for safety evaluation.

\subsubsection{Predictive analysis}

Evidence propagation was conducted to predict the probability distribution of the framework outcome and other relevant variables under the combination of changes in the assumption of certain variables. The aim of the analysis was to predict the probability distribution of the occurrence of pipeline failure factors before an accident occurs. In this section, the propagation of evidence examines several different scenarios and combinations of events taking place (i.e. 100\% probability). To check the proposed Bayesian network model, the percentage of human error in terms of bad response to change, no alarms prioritized and bad quality of data were considered. As a result, the probability of pipeline failure is increases up to approximately $65 \%$ as shown in Figure 6. 


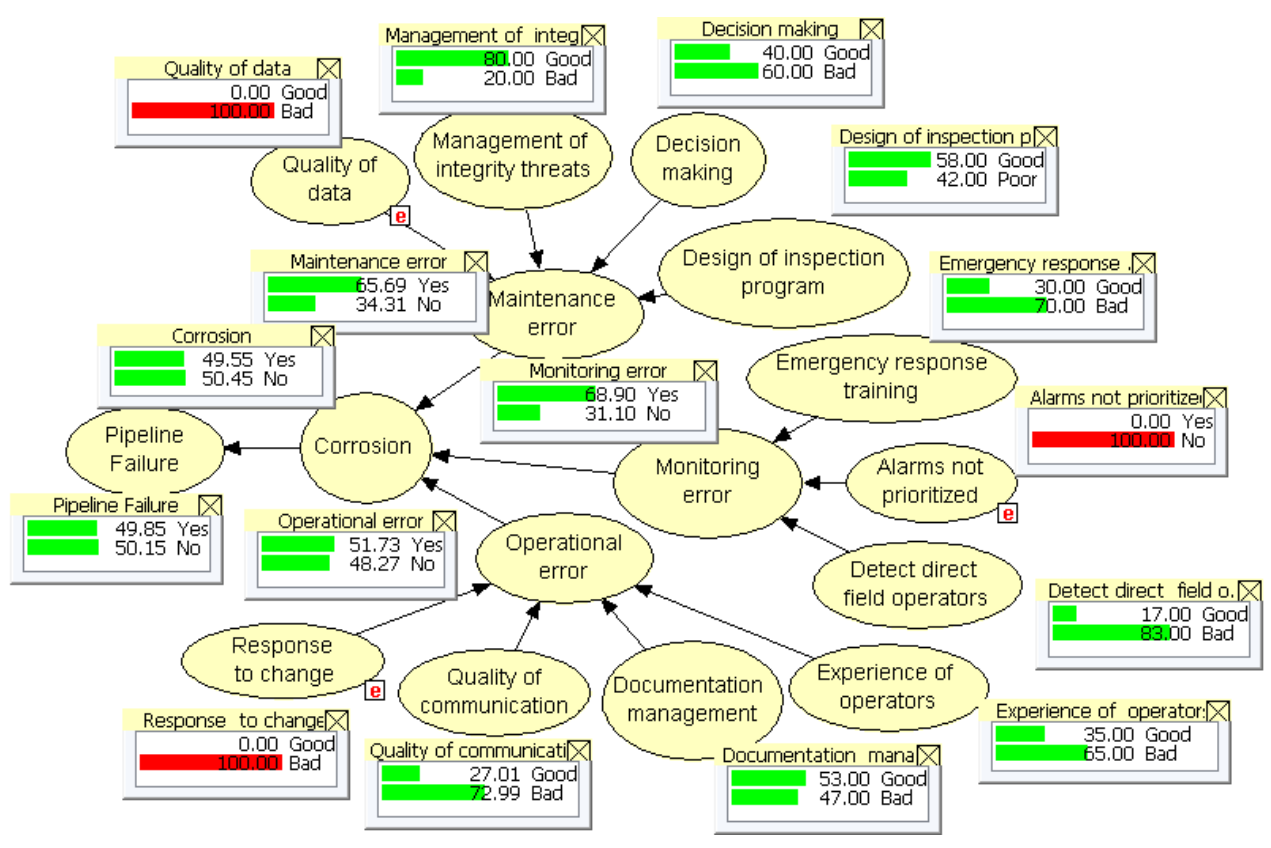

Figure 6: BN Model for Predictive Analysis

According to Bayesian logic, the only method to measure a situation with an undefined outcome is through defining its probability. Bayes' theorem can provide a scientific method that could be used to calculate, given events in prior trials, the probability of a target occurrence in future trials. Hence this Bayesian model predictive-analysis is another example of Bayesian logic which can be used to predict future events for decision making.

\subsection{Counter measures for human error}

From the result obtained, some elements need to be considered in the development of human error management, plan and design include the following:

\subsubsection{Controlling human error in maintenance activities}

- Running a high quality in-line inspection (ILI) tool for the operation of the pipeline. A baseline ILI run could identify construction incidents that need investigation and decide what needs to be done.

- Use of technology to automate repetitive processes that are prone to human error, such as data entry. Automation of some processes could eliminate the potential for copy/paste for human error.

\subsubsection{Controlling human error in monitoring activities}

- Use of expert judgment in rule-based analyses. For example, use of an expert system to evaluate ILI data could reduce the potential for judgment or detecting errors.

- Training personnel on heuristics and biases for much more experience in unexpected situations. 


\subsubsection{Controlling human error in operational activities}

- Training program for decision-making complexity to know how operators will communicate with each other for choosing the best way to handle the problems of the process.

- Experience and knowledge transfer program can reduce human error during unfamiliar tasks, also in communicating for handling sudden changes.

\subsection{CONCLUSIONS}

In this study, a quantitative risk analysis approach due to human error for oil and gas pipelines is constructed using Bayesian network. The proposed model is constructed according to a cause and effect relationship. Two types of analysis were carried out to explore the advantage of the proposed approach. Through forward prediction analysis, the probability of pipeline failure occurrence can be calculated based on the evidence occurred. Meanwhile, through diagnostic analysis, the critical risk factors that may lead to failure occurrence were determined. From the results obtained, the proposed Bayesian network inference techniques can be applied to provide valuable understandings to the prevention of accidents and safety improvement. The counter measure could be suggested based on the results in order to reduce the risk of pipeline failure. In general, this work is mainly based on collection of different existing data sources and the accuracy of the pipeline failure prediction could be further improved with more relevant failure data. As Bayesian Network is able to integrate various type of data, expert judgment together with real data from a specific case study should be utilised for future works.

\section{ACKNOWLEDGEMENT}

The author gratitude goes to the Research and Innovation Department, Universiti Malaysia Pahang, Malaysia, for their support through the RDU1703169 research grant.

\section{REFERENCES}

Adebayo, A., \& Dada, A. S. (2008). An evaluation of the causes of oil pipeline incidents in oil and gas industries in Niger Delta region of Nigeria. Engineering Applied Science,3(3), 279-281.

El-Abbasy, M.S., Senouci, A., Zayed, T., \& Mosleh, F. (2015). A condition assessment model for oil and gas pipelines using integrated simulation and analytic network process. Structure and Infrastructure Engineering, 11(3),263-281.

Hill, B. R. T., Fimeche, B. C., Limited, A. D. L., \& Cb, C. (2012). Pipelines risk analysis. IChemE, 657-670.

Kenett, R. S. (2012). Applications of Bayesian Networks. SSRN Electronic Journal, 116.

Khakzad, N., Khan, F. and Amyotte,P. (2011). Safety analysis in process facilities: Comparison of fault tree and Bayesian network approaches. Reliability Engineering and System Safety, 96, 925-932.

Muhlbauer, W. K., P. (2004). Pipeline Risk Management Manual: Ideas, Techniques, and Resources (3rd ed.). Texas, USA: Elsevier Inc.

Revie, R. W. (2015). Oil and gas pipeline integrity and safety handbook (4th edition), Canada.

Shan, X., Liu, K., \& Sun, P.L. (2017). Risk Analysis on Leakage Failure of Natural Gas 
Pipelines by Fuzzy Bayesian Network with a Bow-Tie Model. Scientific Programming, 2017, 1-21.

Sulaiman, N. S. (2017). Pipeline integrity management system (PIMS) using Bayesian Networks for lifetime extension. Ph.D. dissertation, University of Aberdeen, United Kingdom.

Sulaiman, N. S. \& Tan, H. (2014). Third party damages of offshore pipeline. Journal of Energy Challenges and Mechanics, 1(3), 14-19.

Trucco, P., Cagno, E., Ruggeri, F., Grande, O. (2008). A Bayesian belief network modelling of organisational factors and risk analysis: a case study in maritime transportation. Reliability Engineering and System Safety, 6, 845-856.

Wu, W., Saskatchewan, R., Wai, T., \& Ng, K. (2015). Oil and Gas Pipeline Risk Assessment Model by Fuzzy Inference System and Neural Network. University of Regina Faculty of Graduate Studies and Research Supervisory and Examining Committee.

Weber, P., Medina-Oliva, G., Simon, C., \& Iung, B. (2012). Overview on Bayesian networks applications for dependability, risk analysis and maintenance areas. Engineering Applications of Artificial Intelligence, 25(4), 671-682.

Yang, X. and Mannan, M.S. (2010). The development and application of dynamic operational risk assessment in oil/gas and chemical process industry. Reliability Engineering and System Safety, 95, 806-815.

Yang, X. (2010). The development and application of dynamic operational risk assessment in oil/gas and chemical process industry. Ph.D. dissertation, Texas A\&M University, College Station, TX. 